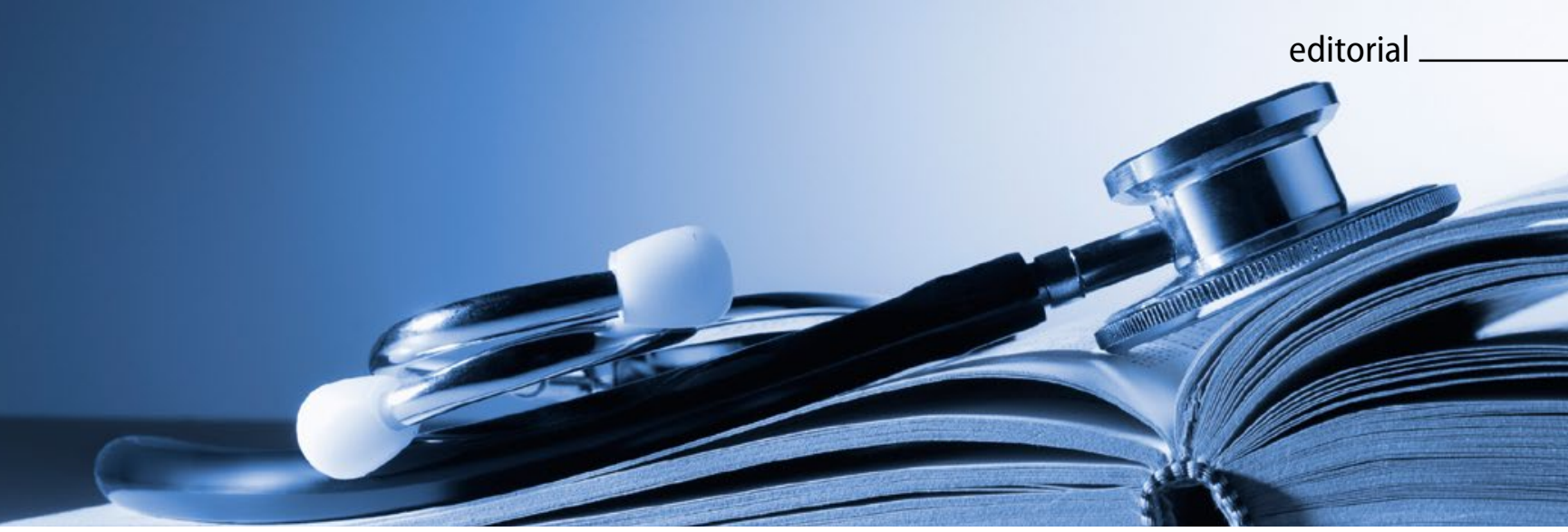

\title{
Neue Zeiten für uns alle!
}

„O tempora, o mores“ äußerte früher der Lateiner, um seinem Erstaunen über neue Zeiten und Herausforderungen Ausdruck zu verleihen. Nachdem die Familie der Dermatologen bei der Fortbildungstagung in München ganz zu Beginn mit den Herausforderungen eines Anschlags bzw. dem Werk eines Irren konfrontiert wurde, fand der Kongress in München dann doch nach zwei Tagen zu seinem gewohnten Verlauf.

In dem Zusammenhang möchte ich den Kongressorganisatoren unter dem Tagungspräsidenten Herrn Professor Ruzicka Dank und Anerkennung aussprechen, die wieder ein hervorragendes Tagungsprogramm zusammengestellt haben. Die Organisation im neuen Kongresszentrum in München war insbesondere von der Industrieausstellung her vorbildlich, die Kommunikation unter den Dermatologen war allerdings an der vorherigen Lokalisation im Gasteig einfacher. Wir werden ja nicht jedes Jahr Umstände wie in 2016 haben, daher gehe ich davon aus, dass auch in der Zukunft die Veranstaltung wieder in gewohnter Manier stattfinden wird. Neben den Biologicals und deren neuesten Entwicklungen spielten auch Laser und weiterentwickelte Geräte zur physikalischen Diagnostik von Hautveränderungen eine gewichtige Rolle im Ausstellungsbereich. Selbstverständlich waren auch die Anbieter von Dermatika gut vertreten.

Die Arbeitsgemeinschaft Ästhetische Dermatologie und Kosmetologie (ADK) e. V. war in den begleitenden Symposien durch mehrere Vorstands- und Beiratsmitglieder aktiv vertreten, einen Seminarblock gestaltete sie sogar selbst. In der Zukunft wollen wir das Wissen im Bereich der ästhetischen und kosmetischen Dermatologie nunmehr klarer gliedern und in Form von Kursen an unsere Mitglieder weitergeben. Der häufig nachgefragte Grundkurs wird zukünftig unter der Leitung von Frau Professorin Borelli in Tübingen stattfinden und über zwei Tage gehen (Freitag und Samstag). Für eine entsprechende Zertifizierung ist gesorgt. Fortgeschrittene Kollegen treffen wir u.a. gerne bei der Tagung Dermatologische Praxis in Frankenthal, wo wir auch im Jahre 2017 wieder ein Symposium am Freitagnachmittag gestalten werden. Durch die Wahl der Termine soll die Möglichkeit sichergestellt sein, dass auch niedergelassene Kollegen jederzeit an unseren Aktivitäten teilnehmen können.

Wir laden Sie schon jetzt dazu ein, uns relevante Themen einzureichen. Selbstverständlich freuen wir uns, wenn aktive Kollegen sich von sich aus als Referenten mit interessanten Themen anbieten. Wichtig wird auch sein, dass wir neue Methoden kritisch in Referaten vorstellen und unsere eigenen Erfahrungen gemeinsam diskutieren. Für unsere Mitglieder haben wir für

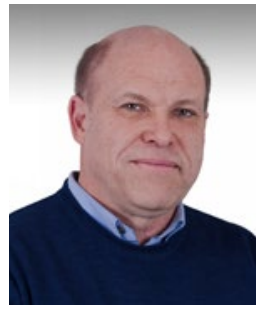

Dr. Matthias Herbst

Generalsekretär ADK e.V.

den Frankenthaler Kongress schon jetzt eine reduzierte Teilnahmegebühr verhandelt, die um mehr als den Jahresbeitrag für die ADK reduziert ist. Eine Mitgliedschaft in der ADK ist also aus mehreren Gründen durchaus sinnvoll. Einen entsprechenden Vordruck zur Anmeldung bei der ADK finden Sie auf Seite 49 in dieser Ausgabe.

In den letzten Tagen haben wir in unserer Praxis verschiedene technische Geräte zur Verbesserung dermatologischer Diagnostik ausprobiert. Es fällt auf, dass die jeweiligen Referenten der Hersteller uns diese Methoden zumeist kompetent präsentieren. Geht man in die Tiefe und fragt nach der Vernetzung der neuen Geräte, zum Beispiel mit der bestehenden Praxisinfrastruktur (Software), so werden im besten Fall noch die GDTSchnittstelle benannt, praktische Erfahrungen aber fehlen. Wir sind in der Praxis zurzeit dabei, entsprechende Erfahrungen zu sammeln, um es uns und den Kollegen zukünftig zu erleichtern, hier zukunftsfähige innovative Lösungen zur Organisation und Dokumentation von Messungen und Befunden in der Praxis zu implementieren. Voraussichtlich können wir mit eServices auch einmal Geld verdienen!? Weitere Einzelheiten werde ich Ihnen gerne bei der Tagung in Frankenthal vorstellen.

Heute wünsche ich Ihnen alles Gute für Ihre Praxis und verbleibe mit den besten Grüßen

Ihr

Dr. Matthias Herbst

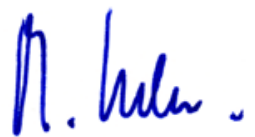

